\title{
An Intermittent Self-Powered Energy Harvesting System From Low-Frequency Hand Shaking
}

\author{
Huicong Liu, Member, IEEE, Zhangping Ji, Tao Chen, Member, IEEE, Lining Sun, \\ Suchith C. Menon, and Chengkuo Lee, Member, IEEE
}

\begin{abstract}
This paper presents the design, fabrication, and characterization of a self-powered energy harvesting system in intermittent working mode. The system consists of an electromagnetic energy harvesting device, a self-driven power management circuit (PMC) and an electronic load. The harvesting device with nonlinear magnetic-spring configuration converts vibration energy into electrical energy from low-frequency hand shaking of human being. The PMC is able to regulate, store the generated energy, and smart control the powering of the electronic load without any external voltage supply. The self-powered energy harvesting system is constructed especially for the circumstance that the power generation is insufficient to directly power-up the load. Future potential application could be self-powered microelectronics and wireless sensor nodes.
\end{abstract}

Index Terms-Self-powered energy harvesting system, low-frequency vibration, self-driven power management circuit.

\section{INTRODUCTION}

$\mathbf{R}$ ECENT advances in microelectronic devices with ultra-low power consumption have stimulated the growing interest of energy harvesting technology [1]-[3]. As the use of conventional batteries has a limited lifetime and may fail at inconvenient time. For example, the autonomous sensor nodes [4] used in the field of environmental monitoring or structure safety detection are numerous and extensively distributed, the replacement of batteries requires plenty of manpower and resources. Energy harvesting technology [5]-[7] could be a promising alternative for reducing the maintenance cost and chemical waste of battery.

Kinetic energy is attractive for the miniature energy harvesting devices, as it exists in a variety of scenarios, such as structural or machines vibrations, and human motions [3]. The devices generate electrical power based on

Manuscript received December 4, 2014; revised January 25, 2015 and March 4, 2015; accepted March 4, 2015. Date of publication March 9, 2015; date of current version July 7, 2015. This work was supported in part by the Foundation Research Project of Jiangsu Province under Grant BK20140335, in part by the National High Technology Research and Development Program (863 Program) of China under Grant 2015AA043502, and in part by the National Natural Science Foundation of China under Grant 51405318. The associate editor coordinating the review of this paper and approving it for publication was Prof. Kazuaki Sawada. (Corresponding author: Tao Chen.)

H. Liu, Z. Ji, T. Chen, and L. Sun are with the Jiangsu Provincial Key Laboratory of Advanced Robotics, Robotics and Microsystems Center, and the Collaborative Innovation Center, Suzhou Nano Science and Technology, Soochow University, Suzhou 215021, China (e-mail: hcliu078@suda.edu.cn; jizhangping@foxmail.com; chent@suda.edu.cn; Insun@suda.edu.cn).

S. C. Menon and C. Lee are with the Department of Electrical and Computer Engineering, National University of Singapore, Singapore 119077 (e-mail: suchithcmenon@gmail.com; elelc@ nus.edu.sg).

Color versions of one or more of the figures in this paper are available online at http://ieeexplore.ieee.org.

Digital Object Identifier 10.1109/JSEN.2015.2411313

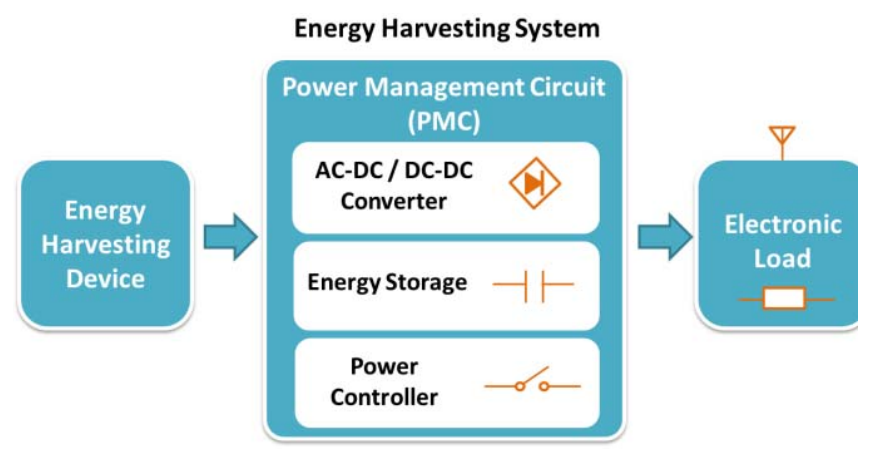

Fig. 1. Schematic diagram of a self-powered energy harvesting system including the energy harvesting device, power management circuit and electronic load.

piezoelectric [8]-[11], electromagnetic [12]-[14] and electrostatic [15], [16] transduction mechanisms. However, in most cases, the generated power may not directly satisfy the electronic devices, because of either the requirement of a constant DC voltage/current or the higher power consumption. Hence, a power processing circuit is necessary and particularly essential as the interface between the harvesting device and the electronic load [17]-[20]. Figure 1 shows a typical energy harvesting system, consisting of an energy harvesting device, a PMC and an electronic load. The PMC should be able to regulate and store the generated energy, and further smart control the powering of the electronic load. In the block diagram of the PMC, an AC-DC converter is designed to accept instantaneous AC voltage in either pulse, periodic or irregular manner, to a steady stream of DC, while a DC-DC converter is selected to steps up or down the DC voltage according to the specification of the storage or load elements. The foremost energy storage devices being considered are microbatteries and supercapacitors. For the circumstance that the generated power is insufficient to directly power-up the electronic load, a power controller is required to switch the circuit in between the charging and discharging modes.

In the literature, various PMCs have been reported for the vibration-based energy harvesting devices. However, a majority of the circuits include only the voltage converter and storage elements. The power control circuit is normally neglected or simplified as a mechanical switch to avoid additional power supply. $\mathrm{Li}$ et al. [21] have reported an electromagnetic energy harvester with management circuit for wireless sensor nodes. Besides the stepping-up and rectifier circuit, the switching capacitor network has been highlighted. 
Through the ON/OFF switching, the capacitors are connected in parallel at charging mode and in series at discharging mode. However, the authors did not discuss the control of the ON/OFF switches, which requires additional control circuit and power consumption. Cao et al. [22] have proposed an energy harvesting circuit with feedforward and feedback control of a DC-DC boost converter. The control circuit is designed to produce desired duty cycle voltage, so as to turn on/off the switches of the converter. However, the control circuit requires external power source and duty cycle voltage to operate the comparator and MOSFET. Similarly, Dayal and Persa [23] and Guo et al. [24] have utilized the AC-DC boost converter or buck-boost converter to amplify the low voltage of the microgenerator. However, the processing circuits require external DC supply to power the gate or MOSFET driver. Amirtharajah and Chandrakasan [25] and Rahimi et al. [26] have presented the self-powered energy harvesting systems. The circuit employed transformers to amplify the low input signals. The transformer must be large enough to achieve the boost effect. Large circuit volume may not suitable for miniature harvesting systems. In addition, the self-powered system requires a backup power source providing a start-up voltage.

In this work, we explore a real sense of self-powering an intermittent microelectronic device by using EMEH device from human motions. The paper describes in detail the design, implementation, and test of the self-powered energy harvesting system. Section II describes a particular electromagnetic nonlinear energy harvesting device to generate power from low-frequency hand shaking. Section III discusses the design of the PMC scheme. Simulation and test results for the overall system are shown in Section IV. Finally, Section V gives concluding remarks on this work and future potential applications.

\section{Electromagnetic Energy Harvesting Device FROM HAND SHAKING VIBRATIONS}

Among the reported vibration-based energy harvesters, most of the devices focus on the power generation in response to harmonic excitations without considering the irregular vibration frequencies occurring in practical applications. Some research groups have made the attempts to conduct the measurements from the real vibration sources. For instance, the harvesting devices have been tested on the bridge, car engine, shoes, and human bags [27]-[29]. Comparing to building and machine vibrations, harvesting energy from human motions are especially challenging because of the ultra-low frequency and irregular vibrations, following by a relatively low power generation. In this work, we use an EMEH device with nonlinear magnetic springs, which offers many advantages of high output performance, ease of construction, less prone to failure and low cost. Most importantly, it is capable of providing considerable power from low-frequency human motions, such as running, walking and hand shaking. Similar prototypes have been reported by several groups from different aspects [30]-[32].

Figure 2(a) illustrates a schematic diagram of the EMEH device with nonlinear magnetic springs. It consists

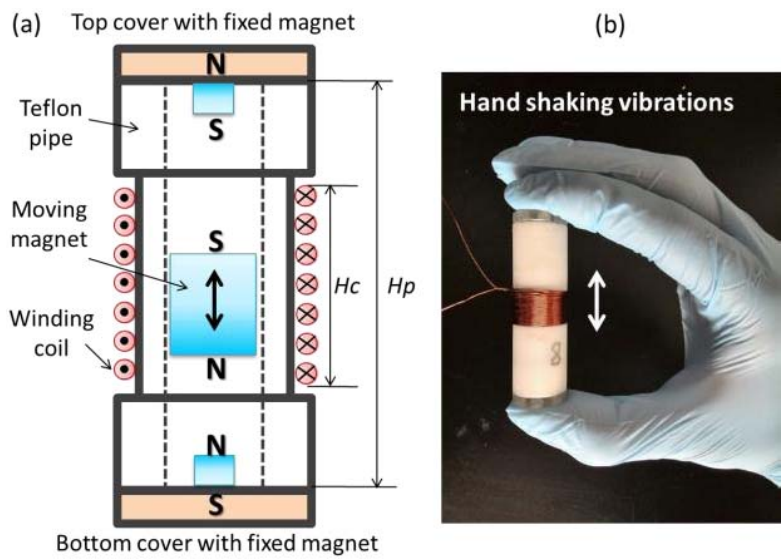

Fig. 2. (a) Schematic diagram and (b) photograph of the EMEH device with nonlinear magnetic springs from hand shaking vibrations.

of a cylindrical pipe made of Teflon, two covers attached with disc magnets at the top and bottom ends of the pipe, a moving bulk magnet inside the pipe, and winding coils made of enamelled copper outside the pipe. The diameter and height of the disc magnets are 3 and $2 \mathrm{~mm}$, respectively, while those of the bulk magnet are 6 and $6 \mathrm{~mm}$, respectively. The three permanent magnets are arranged to make the same magnetic poles face to each other, such that the top and bottom disc magnets serve as magnetic spring to suspend the moving bulk magnet. The copper coils with a total length of $L_{c}$ are wrapped at the middle of the pipe with winding coils height of $H_{C}$ and pipe height of $H_{P}$. The photograph of the EMEH device is shown in Fig. 2(b).

When the pipe vibrates driven by hand shaking, the suspended bulk magnet starts to oscillate through the winding coils. Based on Faraday's law of induction, the change of magnetic flux over the winding coils will be induced and thereby electrical voltage across the coils is generated. The EMEH devices with various parameters are fabricated and their output performances are compared to obtain an optimized device configuration. The varying parameters include pipe height $H_{P}$ of 40,50 and $60 \mathrm{~mm}$, winding coil height $H_{C}$ of 10,15 and $20 \mathrm{~mm}$, coil diameter $d_{C}$ of $0.31,0.14$ and $0.08 \mathrm{~mm}$, and total coil length $l_{C}$ of 10,20 to $90 \mathrm{~m}$. To ensure the comparability of the testing results, the experiments are conducted at relatively constant shaking amplitude of $2 \mathrm{~cm}$ and frequency of 4.5 to $5 \mathrm{~Hz}$. The voltage comparison for each specific parameter is performed on condition that the other parameters remain constant. It is found that a longer pipe length (i.e., $60 \mathrm{~mm}$ ) and a shorter winding coil height (i.e., $10 \mathrm{~mm}$ ) generate more power than the other prototypes. By increasing the pipe length, the magnetic spring constant decreases. Hence, the resonant frequency of the device decreases and gets close to the hand shaking frequency of about $5 \mathrm{~Hz}$. It is known that as the driving frequency approaches to the resonant frequency, the device generates more power. In order to maximize the output voltage for the same total coil length, the winding height of the coil should be shortened such that the magnetic flux is not completely enclosed by the coil over any active generation period. The detailed modeling and optimization 
TABLE I

Parameters of THE Nonlinear EMEH Device

\begin{tabular}{ll}
\hline \hline Parameter & Value \\
\hline Magnetic material & NdFeB $(\mathrm{N} 35)$ \\
Coil material & Copper \\
Housing material & Teflon \\
Young's modulus $(\mathrm{Pa})$ & $1.53 \times 10^{11}$ \\
Coercive Force $(\mathrm{kA} / \mathrm{m})$ & 860 \\
Residual magnetic flux density $(\mathrm{Br})(\mathrm{T})$ & 1.18 \\
Diameter and height of top magnet $(\mathrm{mm})$ & $3 \times 2$ \\
Diameter and height of bottom magnet $(\mathrm{mm})$ & $3 \times 2$ \\
Diameter and height of moving magnet $(\mathrm{mm})$ & $6 \times 6$ \\
Mass of the moving magnet $(m)(\mathrm{g})$ & 1.23 \\
Wire diameter $\left(d_{w}\right)(\mathrm{mm})$ & 0.08 \\
Total wired length $\left(L_{C}\right)(\mathrm{m})$ & 90 \\
Coil width $\left(H_{C}\right)(\mathrm{mm})$ & 10 \\
Pipe length $\left(H_{P}\right)(\mathrm{mm})$ & 60 \\
Inner radius $\left(r_{i n}\right)(\mathrm{mm})$ & 3.5 \\
Outer radius $\left(r_{\text {out }}\right)(\mathrm{mm})$ & 5.5 \\
Magnetic flux density $(B)(\mathrm{T})$ & 0.18 \\
\hline
\end{tabular}

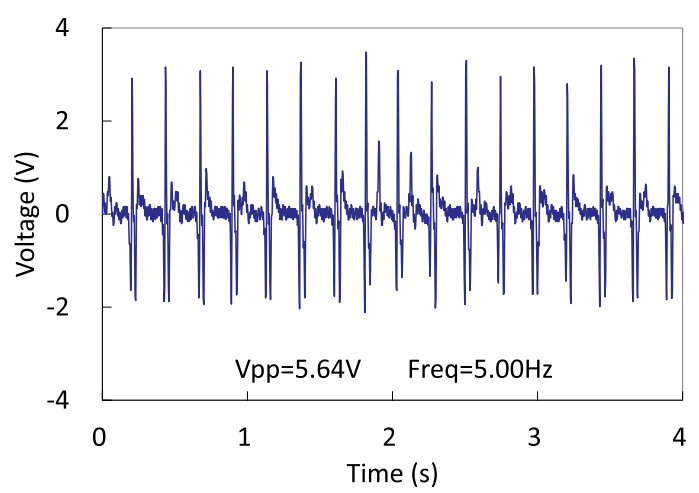

Fig. 3. Output voltage waveform of the optimized EMEH device with pipe length of $60 \mathrm{~mm}$, winding height of $10 \mathrm{~mm}$, coil diameter of $0.08 \mathrm{~mm}$ and total coil length of $90 \mathrm{~m}$.

of this EMEH device can be found in the authors' previous work [33].

According to Kirchhoff's law, the electric current $i$ neglecting coil inductance is given as:

$$
i=\frac{B l(\dot{x}-\dot{z})}{\left(R_{L}+R_{I N}\right)}
$$

where $R_{L}$ and $R_{I N}$ are the load and internal resistances; $l$ is the effective coil length; $B$ is the magnetic flux density of a cylindrical magnet [34]. Based on Eq. (1), the output power of the EMEH device is also dependent on the total coil length or the number of coil turns. The induced electromotive force can be further improved by using longer coil length and thinner wire diameter, at the cost of greater internal coil resistance. For the optimized pipe length of $60 \mathrm{~mm}$ and winding height of $10 \mathrm{~mm}$, the enamelled coil with diameter of $0.08 \mathrm{~mm}$ and total length of $90 \mathrm{~m}$ is designated to wrap around the pipe with 1700 turns. The parameters of the optimized EMEH device are summarized in Table I, and the output spectrum obtained from the oscilloscope is shown in Fig. 3, where the maximum peak-to-peak voltage $V_{p p}$ is achieved to be $5.64 \mathrm{~V}$, and the shaking frequency is around $5 \mathrm{~Hz}$.

\section{Power Management Circuit}

Figure 4 shows a circuit diagram of a self-powered energy harvesting system, where the PMC forms the interface between the harvesting device and the electronic load. The PMC consists of a voltage multiplier \& rectifier module, an energy storage capacitor, a voltage division circuit, a voltage comparator \& controller module, and an analog switch module. The EMEH device produces a time-varying voltage spectrum, but the load circuitry requires a steady DC voltage. Thus, the generated voltage must be regulated and boosted. In addition, the harvesting device is hard to provide enough power in a continuous fashion, as the electronic load may consume more power. A power control circuit is included to charge a storage capacitor and then discharge to the load intermittently. The great strength of the proposed PMC is that it is self-driven by the front-end harvesting device without any external power supply. Even if the harvested power is insufficient, the PMC is still capable of providing an intermittent operating power to the load via a self-driven control circuit.

\section{A. Voltage Multiplier and Storage}

An AC-to-DC converter rectifies the alternative signal delivered by the harvesting device and a DC-to-DC converter adapts the levels of voltage to the storage element and the load. The amount of power that is lost during rectification is mainly due to the high threshold voltage of the diode. A number of active circuits have been proposed to effectively reduce the energy loss, such as adaptive circuits [35], synchronous half-wave and full-wave rectifiers [36], synchronized switching harvesting with inductor (SSHI) technique [37], dual phase charge pump circuit [38], traditional boost and buck converters, etc. However, additional static power is required to operate the circuit. If this power is excessive, it can outweigh the benefits of the reduction in the diode voltage drop.

Besides the above mentioned active rectifying circuits, Villard's voltage multiplier is widely used in cascaded fashion to get high voltage multiplication. The main advantage is that the operation of the passive circuit does not require any external voltage bias. This enables us to generate stand-alone system and is more suitable for ultra-low power applications. As illustrated in Fig. 5, a basic voltage doubler circuit is composed of two rectifier diodes (D1 and D2) and two capacitors ( $\mathrm{C} 1$ and $\mathrm{C} 2$ ). During the positive cycle of the input AC signal $U i$, the current flows through diode D1 while is blocked by diode D2. Thus the voltage $U_{C 1}$ across capacitor $\mathrm{C} 1$ is charged to be $U i\left(U_{C 1}=U i\right)$. During the next negative cycle, the current flows through $\mathrm{D} 2$ while is blocked by $\mathrm{D} 1$. Therefore, the voltage $U_{C 2}$ across the capacitor $\mathrm{C} 2$ is duplicated to be 2 times $U i\left(U_{C 2}=2 U i\right)$, due to the superposition of the input $U i$ and capacitor voltage $U_{C 1}$ in series. In such a way, the voltage multiplier can be constructed by stack multiple voltage doublers in series. It is noted that the amplitude of the input voltage must be greater than the voltage drop of a diode, such that the current can flow through 


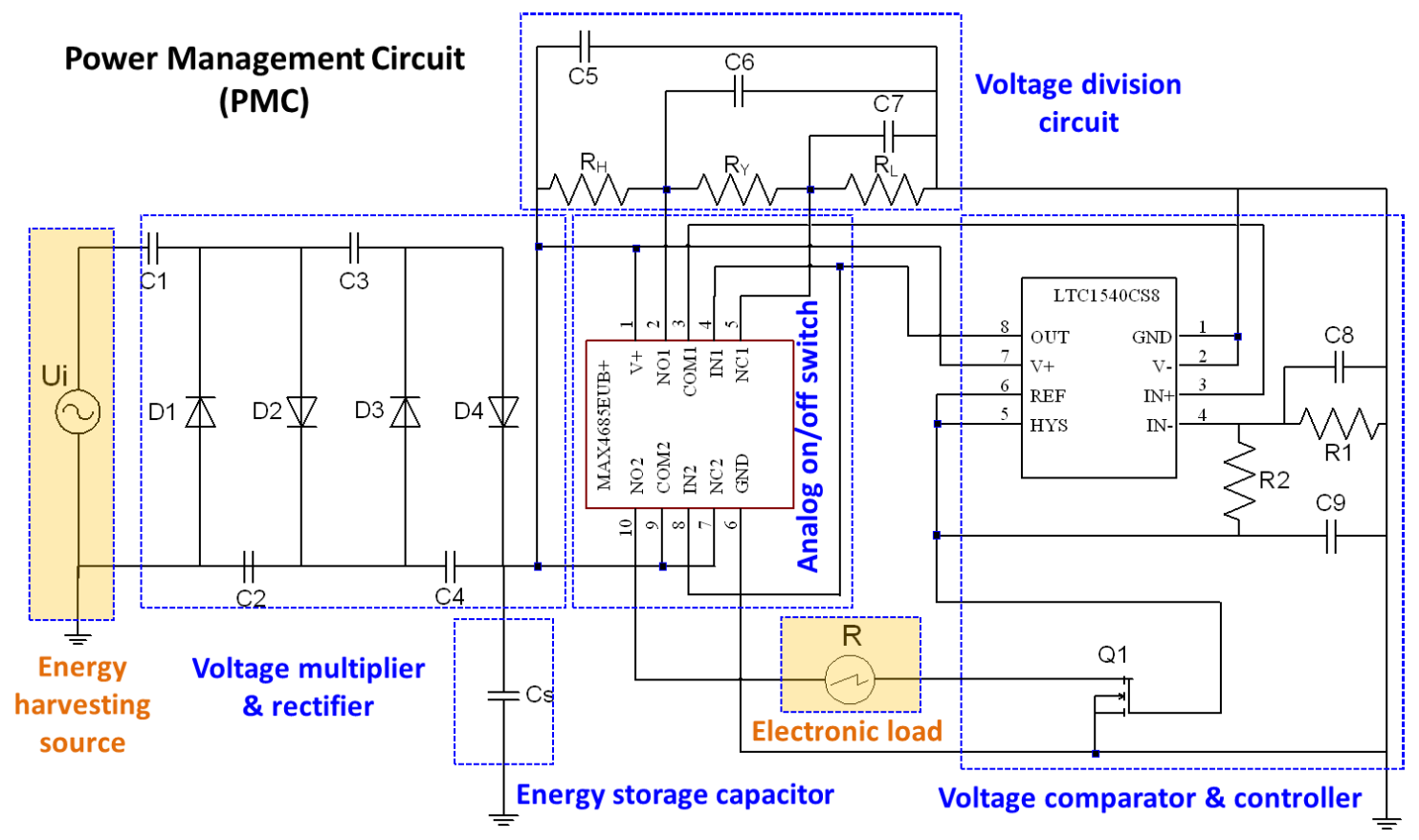

Fig. 4. Circuit diagram of a self-powered energy harvesting system, where the PMC forms the interface between the harvesting device and the electronic load.

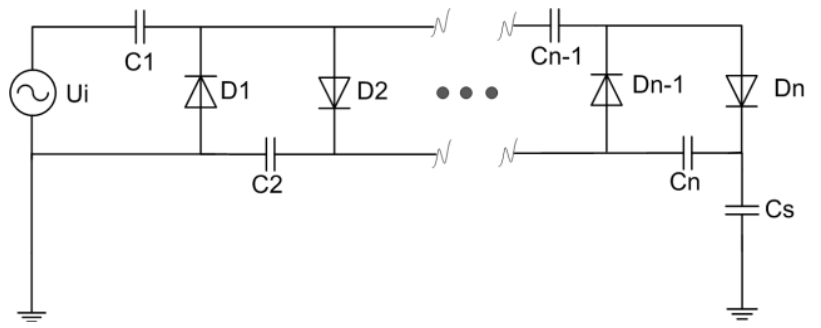

Fig. 5. Circuit diagram of an $\mathrm{n}$-stage voltage multiplier in between an input AC voltage Ui and a storage capacitor Cs.

the diode and the capacitor can be effectively charged for each single stage.

Figure 5 shows an n-stage voltage multiplier. A storage capacitor $\mathrm{Cs}$ is connected to accumulate the increasing electrical energy and later on power the control circuit and load. Theoretically, the rectified saturation voltage $U_{S}$ across the storage capacitor $\mathrm{Cs}$ is $\mathrm{n}$ times of the input peak voltage $U_{i}$ minus the voltage drop of the diode $U_{D}$, which can be expressed as:

$$
U_{S}=n \times\left(U i-U_{D}\right)
$$

The n-stage voltage multiplier circuit is simulated by using SPICE software. The 1N6263 diode models are used in the circuit along with the capacitors of $1 \mu \mathrm{F}$ for all the stages. The 1N6263 schottky diode drop is found to be around $0.15 \mathrm{~V}$. The energy harvesting source is modelled as a sinusoidal voltage waveform with amplitude of $2 \mathrm{~V}$ and frequency of $4 \mathrm{~Hz}$. The storage capacitor is chosen to be $68 \mathrm{uF}$. Figure 6 shows the rectified output voltages across the storage capacitor by the voltage multipliers of 4-stage, 6-stage and 8-stage, respectively. As can be seen, the more stages included in

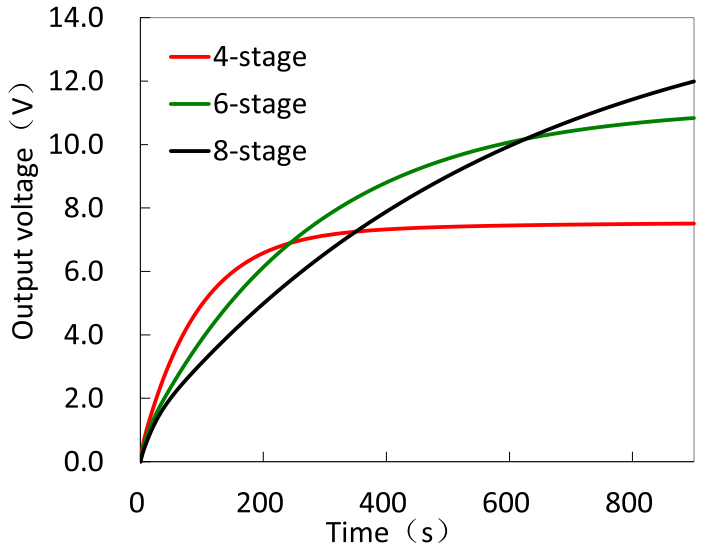

Fig. 6. Rectified output voltages across the storage capacitor by the voltage multipliers of 4-stage, 6-stage and 8-stage, respectively.

the circuit, the higher rectified voltage be achieved, however, at the cost of much longer time to reach the saturation voltage and higher power loss as more components are connected. On the contrary, the voltage multiplier with less stages is able to reach its specific saturation voltage rapidly, which is more conducive to shorten the charging cycle of the capacitor and system response time. Hence, there is a trade-off for the selection of the stage numbers of a voltage multiplier.

\section{B. Voltage Comparator and On/Off Switch Control}

The switch control circuit determines the voltage levels across the storage capacitor $\mathrm{Cs}$ and turns on or off the power supply to the load. To be more specific, when the voltage across Cs reaches an upper pre-determined threshold voltage $\mathrm{V}_{\mathrm{UPPER}}$, the switch is turned on, which enables the 


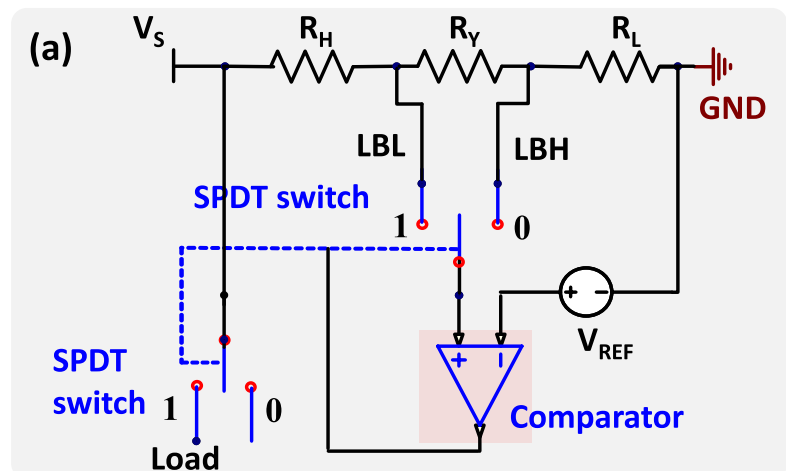

(b) LTC1540

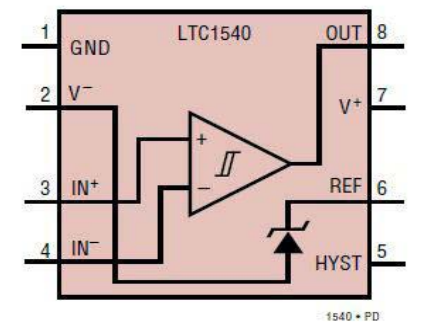

(c) MAX4685

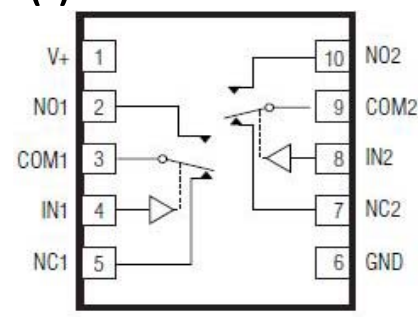

Fig. 7. (a) Schematic diagrams of the switch control circuit, (b) the commercial IC LTC1540 comparator, and (c) the IC MAX4685 SPDT switches.

capacitor to discharge to the load. When the voltage across the discharged capacitor drops below a lower pre-determined threshold voltage $\mathrm{V}_{\text {LOWER }}$, the switch is turned off such that the capacitor stops powering the load. Thereafter, the voltage across the capacitor starts to increase again till it reaches the upper threshold voltage, and the charging and discharging cycles are repeated.

Figure 7(a) shows a schematic diagram of the switch control circuit, which contains a voltage divider, a comparator and a pair of single-pole double-throw (SPDT) switches. Figure 7(b) and (c) shows respectively a commercial integrated circuit (IC) LTC1540 serves as the comparator and the IC MAX4685 integrates a pair of analog switches. The LTC1540 is an ultra-low power, single comparator with builtin reference $\mathrm{V}_{\mathrm{REF}}$ of $1.182 \mathrm{~V}$. The comparator operates from a $2 \mathrm{~V}$ to $11 \mathrm{~V}$ single supply as long as connecting V- to GND. The absolute maximum rated voltage parameters of $\mathrm{IN}^{+}, \mathrm{IN}^{-}$, HYST are $-0.3 \mathrm{~V}$ to $\left(0.3 \mathrm{~V}+\mathrm{V}^{+}\right)$, and the maximum rated current parameters of $\mathrm{IN}^{+}, \mathrm{IN}^{-}$, HYST are $20 \mathrm{~mA}$. Operation below $1.5 \mathrm{~V}$ is not recommended because of the increase in supply current. The MAX4685 are low on-resistance, low-voltage, dual SPDT analog switches that operate from a $1.8 \mathrm{~V}$ to $5.5 \mathrm{~V}$ supply. The device has break-before-make switching and fast switching speeds $\left(\mathrm{t}_{\mathrm{ON}}=50 \mathrm{~ns} \max\right.$, $\left.\mathrm{t}_{\mathrm{OFF}}=40 \mathrm{~ns} \max \right)$. The absolute maximum rated voltage parameters of $\mathrm{V}^{+}, \mathrm{IN}_{-}$are $-0.3 \mathrm{~V}$ to $+6 \mathrm{~V}$ and $\mathrm{COM}_{-}$, $\mathrm{NO}_{-}, \mathrm{NC}_{-}$are $-0.3 \mathrm{~V}$ to $\left(0.3 \mathrm{~V}+\mathrm{V}^{+}\right)$. The $\max -$ imum rated current parameters of $\mathrm{COM}_{-}, \mathrm{NO}_{-}, \mathrm{NC}_{-}$ are $\pm 300 \mathrm{~mA}$.

The LTC1540 provides a fixed reference voltage $V_{\text {REF }}$ in its pin no. 6 , which is connected to $\mathrm{IN}^{-}$(pin no. 4). Three resistors $\mathrm{R}_{\mathrm{H}}, \mathrm{R}_{\mathrm{Y}}$ and $\mathrm{R}_{\mathrm{L}}$ in series connection are functioned as a voltage divider. Vs is the voltage across the storage capacitor.
The voltage across $\mathrm{R}_{\mathrm{L}}$ and the sum of the voltage across $\mathrm{R}_{\mathrm{L}}$ and $\mathrm{R}_{\mathrm{Y}}$ are connected to the $\mathrm{IN}^{+}$(pin no. 3) so as to compare with the reference voltage output $\mathrm{V}_{\mathrm{REF}}$. By means of setting the resistances $R_{H}, R_{Y}$ and $R_{L}$, the voltage divider determines the upper and lower threshold voltages according to the following equations:

The upper threshold voltage $\mathrm{V}_{\text {UPPER }}$ :

$$
V_{U P P E R}=\frac{V_{R E F} \times\left(R_{H}+R_{Y}+R_{L}\right)}{R_{L}}
$$

The lower threshold voltage $\mathrm{V}_{\text {LOWER }}$ :

$$
V_{L O W E R}=\frac{V_{R E F} \times\left(R_{H}+R_{Y}+R_{L}\right)}{R_{Y}+R_{L}}
$$

The output signal (pin no. 8) of the comparator is connected to pin no. 4 and 8 of the IC MAX4685, which determines the ON/OFF states of the SPDT switches. When the storage capacitor is in charging mode, the output signal of the comparator is " 0 " and both of the SPDT switches are positioned at " 0 " ends. Hence, the switch turns off the power supply of the capacitor to the load and the non-inverting pin of the comparator is connected to $\mathrm{LBH}$. When the voltage of the capacitor in charging mode increases to $\mathrm{V}_{\mathrm{UPPER}}$, the output signal of the comparator becomes " 1 ". Therefore, the power supply to the load is switched $\mathrm{ON}$ and the non-inverting pin is connected to LBL. At this moment, the storage capacitor is in discharging mode. The voltage Vs drops significantly to a lower threshold voltage VLOWER, which leads to a "0" output signal of the comparator. The SPDT switches are triggered to OFF state again. In such a way, the ON/OFF switch control is repeated. It should be noted that the charging/discharging of the capacitor is realized by the self-driven control circuit without any external power source.

\section{Simulation of the PMC}

By using SPICE software, the PMC in Fig. 4 is simulated. The energy harvesting source is assumed to be a sinusoidal voltage with amplitude of $2 \mathrm{~V}$ and frequency of $4 \mathrm{~Hz}$. The electronic load in the circuit is simplified as a lightemitting diode (LED). The upper and lower threshold voltages (i.e., $\mathrm{V}_{\text {UPPER }}$ and $\mathrm{V}_{\text {LOWER }}$ ) are determined by the resistances $\mathrm{R}_{\mathrm{H}}, \mathrm{R}_{\mathrm{Y}}$ and $\mathrm{R}_{\mathrm{L}}$, which are chosen as $20 \mathrm{M} \Omega, 3.9 \mathrm{M} \Omega$ and 4.7 $\mathrm{M} \Omega$, respectively. The reference voltage provided by the comparator is $1.182 \mathrm{~V}$ with respect to $\mathrm{V}-$. In this work, resistors of $\mathrm{R} 1=\mathrm{R} 2=20 \mathrm{M} \Omega$ are used to divide the $\mathrm{V}_{\mathrm{REF}}$ to be $0.591 \mathrm{~V}$. As long as the $\mathrm{V}_{\text {UPPER }}$ and $\mathrm{V}_{\text {LOWER }}$ are determined, the value of $\mathrm{V}_{\mathrm{REF}}$ to be 1.182 or 0.591 does not affect the results. By substituting these values of resistances and $\mathrm{V}_{\mathrm{REF}}=0.591 \mathrm{~V}$ in Eqs. (3) and (4), the $\mathrm{V}_{\text {UPPER }}$ and $\mathrm{V}_{\text {LOWER }}$ are obtained to be 3.60 and $1.97 \mathrm{~V}$, respectively. Hence, the LED is able to operate in between the threshold voltages. From Fig. 6, 4-stage voltage multiplier is selected due to its fast charging time and relatively low power loss.

Figure 8 shows the simulated voltage waveform across the storage capacitor of $100 \mu \mathrm{F}$ (Fig. 8(a)) and $68 \mu \mathrm{F}$ (Fig. 8(c)) in red, meanwhile, the corresponding currents through the LED in blue (Figs. 8(b) and (d)). As the energy harvesting source charges up the storage capacitor constantly, the voltage 


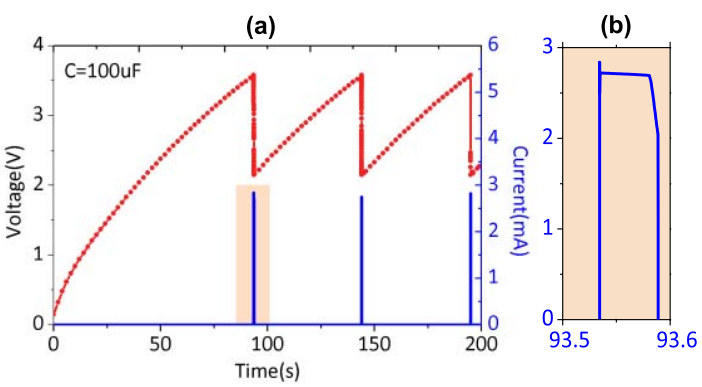

(c)

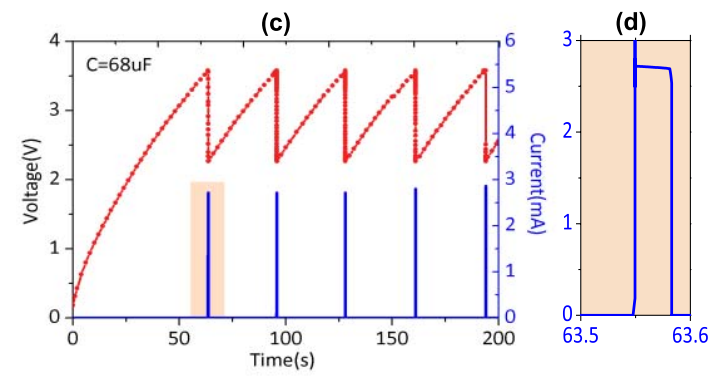

Fig. 8. Simulated voltage waveform across the storage capacitor of $100 \mu \mathrm{F}$ (a) and $68 \mu \mathrm{F}$ (c) in red, meanwhile, the corresponding currents through the LED in blue ((b) and (d)).

across the capacitor rises continuously to the upper threshold voltage of $3.60 \mathrm{~V}$. At this moment, the SPDT switches in the IC MAX4685 are triggered to turn on and the capacitor starts to discharge to the LED. It is obvious that the voltage across the capacitor drops dramatically to the lower threshold voltage of about $2.2 \mathrm{~V}$. Henceforward, the voltage rises during the charging period and drops during the discharging period at regular time intervals. It is seen that the lower threshold voltage is not exactly matches the calculated $\mathrm{V}_{\text {LOWER }}$ of $1.97 \mathrm{~V}$. Because in the simulation a resistor of $200 \Omega$ is connected in between the MOSFET and electronic load, so as to obtain a relatively steady current through the electronic load during the discharging period. The blue curve shows the corresponding current pulse through the LED. The LED current is turned on and off at regular intervals depending on the output voltage of the capacitor. The ON time equals the discharging time of the capacitor while the OFF time equals the charging time. The ON current of a LED is equal to $2.7 \mathrm{~mA}$. The charging and discharging of the capacitor is essentially a process of accumulation and dissipation of electric charges on the electrodes of the capacitor. It is found from Figs. 8(a) and (c) that, the initial charging time for capacitances of 68 and $100 \mu \mathrm{F}$ are 63.55 and $93.53 \mathrm{~s}$, respectively. The regular charging and discharging intervals are characterized as 32.2 and $0.03 \mathrm{~s}$ for capacitance of $68 \mu \mathrm{F}$, and 50.7 and $0.05 \mathrm{~s}$ for capacitance of $100 \mu \mathrm{F}$. Comparing with the small capacitor of $68 \mu \mathrm{F}$, the larger one of $100 \mu \mathrm{F}$ has longer charging and discharging time to accumulate and dissipate the electric charges.

The rate of charge of the capacitor depends on the product of $\mathrm{R}$ and $\mathrm{C}$, which is called the time constant and denoted as $\tau=\mathrm{R}$. C. Figure 9 shows the ON current of different load resistances, i.e., R1 $=2000 \Omega$, R2 $=1000 \Omega$ and $\mathrm{R} 3=500 \Omega$, for a constant storage capacitance of $100 \mu \mathrm{F}$.

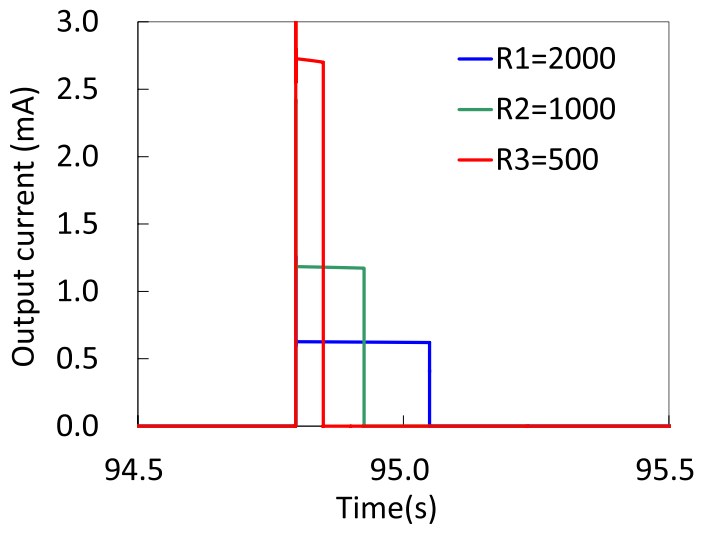

Fig. 9. ON current of different load resistances of $\mathrm{R} 1=2000 \Omega$, $\mathrm{R} 2=1000 \Omega$ and $\mathrm{R} 3=500 \Omega$, for a constant storage capacitance of $100 \mu \mathrm{F}$

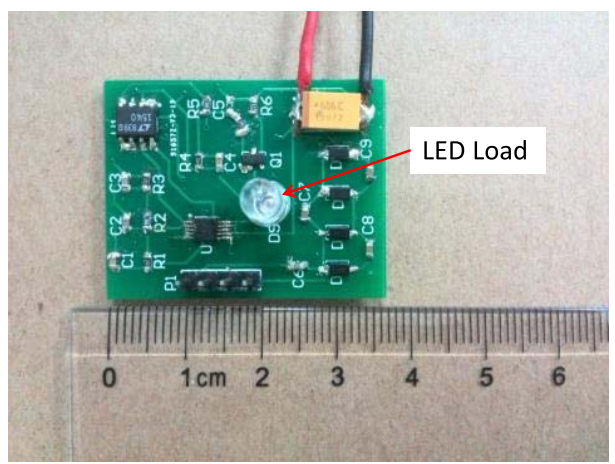

Fig. 10. Photograph of a PCB board integrated with the PMC and LED load with a small size of $1160 \times 1465$ mils.

As can be seen, the larger the resistance, the longer the discharging time, at the cost of a lower ON current. On the contrary, a smaller value resistor induces a shorter discharging time but much higher ON current flow.

\section{Test Results of the SElf-Powered ENERGY HARVESTING SYSTEM}

To demonstrate the feasibility of a self-powered energy harvesting system, the EMEH device, the PMC and the electronic load are constructed. The electronic components and layout of the PMC are integrated onto a PCB board of size of $1160 \times 1465$ mils, as shown in Fig. 10. The electronic load is simplified as a LED, which is selected for easy observing the working state of the load. In the PMC, the upper and lower threshold voltages are set as 3.60 and $1.97 \mathrm{~V}$, respectively. To reach the upper threshold voltage rapidly, a 4-stage voltage amplifier and a storage capacitor of $68 \mu \mathrm{F}$ are incorporated on the PCB board. The whole self-powered system is tested using the optimized energy harvesting pipe from hand shaking. An oscilloscope is connected across the storage capacitor of the circuit to monitor the charging and discharging states of the capacitor via the voltage output.

Figure 11 shows the output voltage across the storage capacitor from the optimized EMEH pipe by hand-shaking, where the generated voltage waveform can refer to Fig. 3. It is observed that the voltage across the capacitor increases 


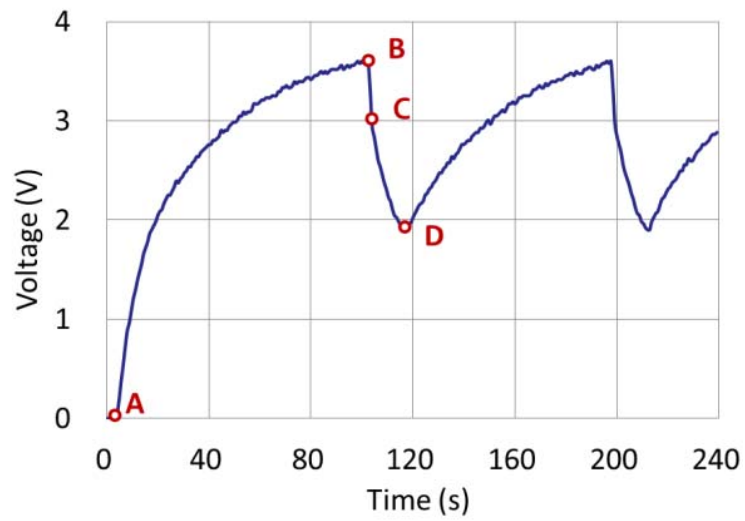

Fig. 11. Output voltage across the storage capacitor from the optimized EMEH device by hand-shaking.

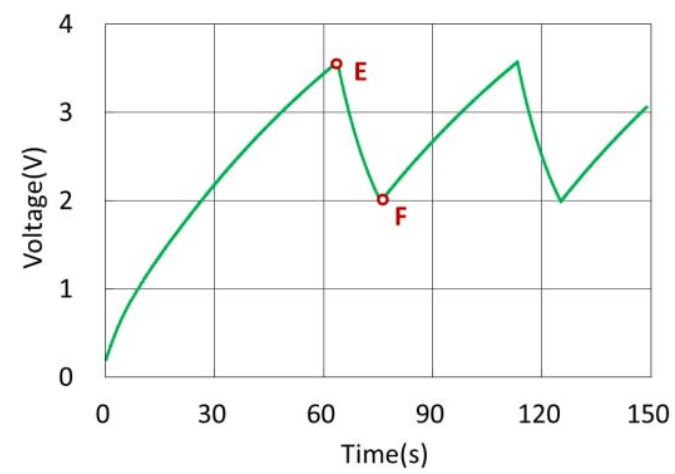

Fig. 12. Simulation result of the output voltage across the storage capacitor by using a resistance load of $200 \mathrm{~K} \Omega$.

from $0 \mathrm{~V}$ at point $\mathrm{A}$ to $3.6 \mathrm{~V}$ at point $\mathrm{B}$ gradually after $103 \mathrm{~s}$. At this moment, the control switch is turned on and the capacitor starts to power up the load. The LED is lightening up spontaneously. Then, the voltage across the capacitor drops dramatically from $3.6 \mathrm{~V}$ at point $\mathrm{B}$ to $3 \mathrm{~V}$ at point $\mathrm{C}$. Henceforward, the voltage is observed to decrease gradually from point $\mathrm{C}$ to $\mathrm{D}$ for $15 \mathrm{~s}$, which does not perfectly match the simulation results in Fig. 8(c). This is due to the sudden decrease of the LED current and hence an extremely large resistance of the LED is observed. According to the forwardbiased IV diagram of a diode, the current is initially very low and rise very slowly. As the forward bias reaches to the ON voltage, the current rises exponentially with a small bias. From Fig. 11, as the voltage decrease from point $\mathrm{B}(3.6 \mathrm{~V})$ to $\mathrm{C}(3 \mathrm{~V})$, the current through the LED experiences a significant drop. From the $\mathrm{ON}$ voltage at point $\mathrm{C}$, there is still a small current flowing through the LED. Hence, a tiny soft light can be observed due to the persistent phosphors of the LED. From point $\mathrm{C}$ to $\mathrm{D}$, the resistance of the LED is quite large of about $200-220 \mathrm{~K} \Omega$, or even up to $1 \mathrm{M} \Omega$. Based on the time constant of a capacitor, i.e., $\tau=\mathrm{R} \cdot \mathrm{C}$, a larger resistance results in a longer discharging time. Therefore, the capacitor is able to illuminate the LED for $15 \mathrm{~s}$. To verify the explanation, the LED load is replaced by a resistance load of $200 \mathrm{~K} \Omega$ in the PMC simulation. It is obvious from Fig. 12 that the discharging time is greatly increased from $64 \mathrm{~s}$ at point $\mathrm{E}$ to $76 \mathrm{~s}$ at point $\mathrm{F}$. From point $\mathrm{D}$, a second round of charging and discharging of the capacitor is followed through the ON/OFF switch. Comparing with the simulation results in Fig. 8(c), the tested circuit requires higher voltage source and longer time to reach the discharging mode. This is because the power consumption of the system is induced not only by the LED load but also by the testing oscilloscope. Without the connection of the oscilloscope, it is found a shorter charging time of 50s from point A to B can be achieved, which is coincide with the simulation.

\section{CONCLUding REMARKS}

This work presents the design, implementation and test of a self-powered energy harvesting system. By using a nonlinear EMEH device from low-frequency hand-shaking and a self-driven PMC, the system is demonstrated to self-power an intermittent microelectronic load. The EMEH device utilizes magnetic-spring to harvest the low-frequency hand shaking energy of $4.5-5 \mathrm{~Hz}$ and generates maximum peak-to-peak voltage of $5.64 \mathrm{~V}$. Without any external source, the self-driven PMC is designed to regulate and accumulate the generated electrical energy to a storage capacitor, and self-control the charging and discharging of the capacitor. For the scenario that power generation from environmental vibration is insufficient or unstable to directly power-up the electronic load, it provides a good solution of the selfpowered microelectronics and wireless sensor nodes in the intermittent working mode. For example, wireless sensor node for measuring the human physical parameters may not require a continuous real-time monitoring. An interval charging of several mins is satisfied. In the future, the PMC can be integrated with other types of energy harvesting devices and sensor nodes for wide application, such as environment surveillance, human body and structural health monitoring.

\section{REFERENCES}

[1] P. D. Mitcheson, E. M. Yeatman, G. K. Rao, A. S. Holmes, and T. C. Green, "Energy harvesting from human and machine motion for wireless electronic devices," Proc. IEEE, vol. 96, no. 9, pp. 1457-1486, Sep. 2008.

[2] J. A. Paradiso and T. Starner, "Energy scavenging for mobile and wireless electronics," IEEE Pervasive Comput., vol. 4, no. 1, pp. 18-27, Jan./Mar. 2005.

[3] S. Roundy, P. K. Wright, and J. Rabaey, "A study of low level vibrations as a power source for wireless sensor nodes," Comput. Commun., vol. 26 , no. 11, pp. 1131-1144, Jul. 2003.

[4] D. Diamond, S. Coyle, S. Scarmagnani, and J. Hayes, "Wireless sensor networks and chemo-/biosensing," Chem. Rev., vol. 108, no. 2, pp. 652-679, 2008.

[5] K. A. Cook-Chennault, N. Thambi, and A. M. Sastry, "Powering MEMS portable devices - A review of non-regenerative and regenerative power supply systems with special emphasis on piezoelectric energy harvesting systems," Smart Mater. Struct., vol. 17, no. 4, p. 043001, 2008.

[6] D. Guyomar et al., "Synchronized switch harvesting applied to selfpowered smart systems: Piezoactive microgenerators for autonomous wireless transmitters," Sens. Actuators A, Phys., vol. 138, no. 1, pp. 151-160, 2007.

[7] E. P. James et al., "An investigation of self-powered systems for condition monitoring applications," Sens. Actuators A, Phys., vol. 110 nos. 1-3, pp. 171-176, 2004.

[8] S. R. Anton and H. A. Sodano, "A review of power harvesting using piezoelectric materials (2003-2006)," Smart Mater. Struct., vol. 16, no. 3, pp. R1-R21, 2007. 
[9] H. Liu, T. Chen, C. Lee, T. Kobayashi, and S. Zhang, "Flow sensing and energy harvesting characteristics of a wind-driven piezoelectric $\mathrm{Pb}\left(\mathrm{Zr} 0.52\right.$, Ti0.48) $\mathrm{O}_{3}$ microcantilever," IET Micro Nano Lett., vol. 9 , no. 4, pp. 286-289, 2014

[10] H. Liu, C. J. Tay, C. Quan, T. Kobayashi, and C. Lee, "Piezoelectric MEMS energy harvester for low-frequency vibrations with wideband operation range and steadily increased output power," J. Microelectromech. Syst., vol. 20, no. 5, pp. 1131-1142, Oct. 2011

[11] Y. Feng, K. Hagiwara, Y. Iguchi, and Y. Suzuki, "Trench-filled cellular parylene electret for piezoelectric transducer," Appl. Phys. Lett., vol. 100, no. 26, p. 262901, 2012.

[12] S. P. Beeby et al., "A micro electromagnetic generator for vibration energy harvesting," J. Micromech. Microeng., vol. 17, no. 7, pp. 1257-1265, 2007.

[13] H. Liu, K. H. Koh, and C. Lee, "Ultra-wide frequency broadening mechanism for micro-scale electromagnetic energy harvester," Appl. Phys. Lett., vol. 104, no. 5, p. 053901, 2014.

[14] B. Yang et al., "Electromagnetic energy harvesting from vibrations of multiple frequencies," J. Micromech. Microeng., vol. 19, no. 3, p. 035001, 2009.

[15] D. S. Nguyen, E. Halvorsen, G. U. Jensen, and A. Vogl, "Fabrication and characterization of a wideband MEMS energy harvester utilizing nonlinear springs," J. Micromech. Microeng., vol. 20, no. 12, p. 125009 , 2010.

[16] Y. Suzuki, "Recent progress in MEMS electret generator for energy harvesting," IEEJ Trans. Elect. Electron. Eng., vol. 6, no. 2, pp. 101-111, 2011.

[17] T. Jirků, P. Fiala, and M. Kluge, "Magnetic resonant harvesters and power management circuit for magnetic resonant harvesters," Microsyst. Technol., vol. 16, no. 5, pp. 677-690, 2010.

[18] M. Lallart, E. Lefeuvre, C. Richard, and D. Guyomar, "Self-powered circuit for broadband, multimodal piezoelectric vibration control," Sens. Actuators A, Phys., vol. 143, no. 2, pp. 377-382, May 2008.

[19] A. M. Paracha, P. Basset, D. Galayko, F. Marty, and T. Bourouina, "A silicon MEMS DC/DC converter for autonomous vibration-toelectrical-energy scavenger," IEEE Electron Device Lett., vol. 30, no. 5, pp. 481-483, May 2009.

[20] C. Peters, D. Spreemann, M. Ortmanns, and Y. Manoli, "A CMOS integrated voltage and power efficient AC/DC converter for energy harvesting applications," J. Micromech. Microeng., vol. 18, no. 10, p. 104005 , Oct. 2008

[21] P. Li, Y. Wen, P. Liu, X. Li, and C. Jia, "A magnetoelectric energy harvester and management circuit for wireless sensor network," Sens. Actuators A, Phys., vol. 157, no. 1, pp. 100-106, 2010.

[22] X. Cao, W.-J. Chiang, Y.-C. King, and Y.-K. Lee, "Electromagnetic energy harvesting circuit with feedforward and feedback DC-DC PWM boost converter for vibration power generator system," IEEE Trans. Power Electron., vol. 22, no. 2, pp. 679-685, Mar. 2007.

[23] R. Dayal and L. Persa, "Low-power low-voltage AC-DC converters for electromagnetic energy harvesting with efficient indirect feedback scheme," IET Power Electron., vol. 5, no. 9, pp. 1923-1933, Nov. 2012.

[24] T. Guo, R. Kerley, and D. S. Ha, "Development of a power conditioning circuit for railcar energy harvesting," in Proc. IEEE Int. Midwest Symp. Circuits Syst., Aug. 2013, pp. 513-516.

[25] R. Amirtharajah and A. P. Chandrakasan, "Self-powered signal processing using vibration-based power generation," IEEE J. Solid-State Circuits, vol. 33, no. 5, pp. 687-695, May 1998.

[26] A. Rahimi, Ö. Zorlu, A. Muhtaroğlu, and H. Külah, "An electromagnetic energy harvesting system for low frequency applications with a passive interface ASIC in standard CMOS," Sens. Actuators A, Phys., vol. 188, pp. 158-166, Dec. 2012.

[27] P. Glynne-Jones, M. J. Tudor, S. P. Beeby, and N. M. White, "An electromagnetic, vibration-powered generator for intelligent sensor systems," Sens. Actuators A, Phys., vol. 110, nos. 1-3, pp. 344-349, Feb. 2004.

[28] T. V. Galchev, J. McCullagh, R. L. Peterson, and K. Najafi, "Harvesting traffic-induced vibrations for structural health monitoring of bridges," J. Micromech. Microeng., vol. 21, no. 10, p. 104005, 2011.

[29] V. Bedekar, J. Oliver, and S. Priya, "Pen harvester for powering a pulse rate sensor," J. Phys. D, Appl. Phys., vol. 42, no. 10, p. 105105, 2009.

[30] C. R. Saha, T. O'Donnell, N. Wang, and P. McCloskey, "Electromagnetic generator for harvesting energy from human motion," Sens. Actuators A Phys., vol. 147, no. 1, pp. 248-253, 2008.

[31] E. Dallago, M. Marchesi, and G. Venchi, "Analytical model of a vibrating electromagnetic harvester considering nonlinear effects," IEEE Trans. Power Electron., vol. 25, no. 8, pp. 1989-1997, Aug. 2010.
[32] A. R. M. Foisal, C. Hong, and G.-S. Chung, "Multi-frequency electromagnetic energy harvester using a magnetic spring cantilever," Sens. Actuators A, Phys., vol. 182, pp. 106-113, Aug. 2012.

[33] H. Liu, S. Gudla, F. A. Hassani, C. H. Heng, Y. Lian, and C. Lee, "Investigation of the nonlinear electromagnetic energy harvesters from hand shaking," IEEE Sensors J., vol. 15, no. 4, pp. 2356-2364, Apr. 2015.

[34] T. Zhang, C. Jiang, H. Xu, and J. Mao, "Permanent-magnet longitudinal fields for magnetostrictive devices," J. Appl. Phys., vol. 101, no. 3, p. $034511,2007$.

[35] G. K. Ottman, H. F. Hofmann, A. C. Bhatt, and G. A. Lesieutre, "Adaptive piezoelectric energy harvesting circuit for wireless remote power supply," IEEE Trans. Power Electron., vol. 17, no. 5, pp. 669-676, Sep. 2002.

[36] T. T. Le, J. Han, A. von Jouanne, K. Mayaram, and T. S. Fiez, "Piezoelectric micro-power generation interface circuits," IEEE J. Solid-State Circuits, vol. 41, no. 6, pp. 1411-1420, Jun. 2006

[37] E. Lefeuvre, A. Badel, C. Richard, L. Petit, and D. Guyomar, "A comparison between several vibration-powered piezoelectric generators for standalone systems," Sens. Actuators A, Phys., vol. 126, no. 2, pp. 405-416, 2006.

[38] P. C.-P. Chao, C. I. Shao, C. X. Lu, and C. K. Sung, "A new energy harvest system with a hula-hoop transformer, micro-generator and interface energy-harvesting circuit," Microsyst. Technol., vol. 17, nos. 5-7, pp. 1025-1036, 2011.

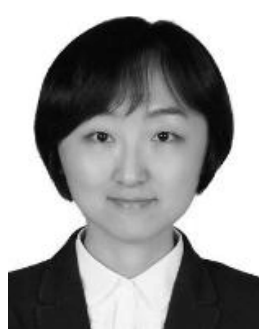

Huicong Liu received the B.Eng. and M.Sc. degrees from the Department of Mechanical Engineering, University of Science and Technology Beijing, China, in 2006 and 2008, respectively, and the Ph.D. degree from the Department of Mechanical Engineering, National University of Singapore (NUS), in 2013. She was a Research Fellow with the Department of Electrical and Computer Engineering, NUS, from 2012 to 2013. She is currently an Associate Professor with the Robotics and Microsystems Center, School of Mechanical and Electrical Engineering, Soochow University, China. Her research interests are vibration-based microelectromechanical systems (MEMS)/nanoelectromechanical systems energy harvesters and self-powered MEMS devices and systems.

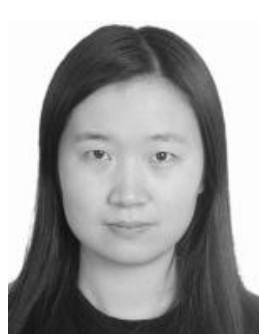

Zhangping Ji received the B.Eng. degree from the Department of Electrical Engineering and Automation, Soochow University, Suzhou, China, in 2014. She is currently pursuing the master's degree at the Robotics and Microsystems Center, School of Mechanical and Electrical Engineering, Soochow University. Her research interests are microelectromechanical systems (MEMS) energy harvesters and self-powered MEMS systems for wireless sensor networks.

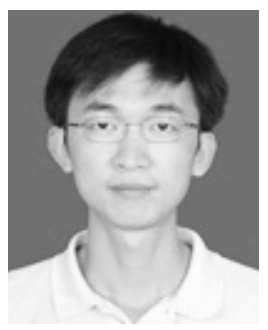

Tao Chen received the bachelor's and master's degrees in mechatronics engineering from the Harbin Institute of Technology, China, in 2004 and 2006, respectively, and the Ph.D. degree in mechatronics from the Harbin Institute of Technology. He is currently an Associate Professor with the Robotics and Microsystems Center, School of Mechanical and Electrical Engineering, Soochow University, China. His research interests are microelectromechanical systems (MEMS) 3-D assembly and micromanipulation robot, including mechanical analysis and material characterization in MEMS, system dynamics and control of mechatronics and MEMS, and electrostatically actuated systems. 


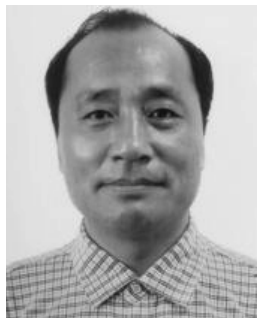

Lining Sun received the bachelor's degree in mechanical engineering and the master's degree from the Harbin Institute of Technology, in 1985 and 1988, respectively, and the Ph.D. degree in mechatronics engineering, in 1993. He is currently a Professor with the Robotics and Microsystems Center, School of Mechanical and Electrical Engineering, Soochow University, China. $\mathrm{He}$ has authored over 300 academic papers, and holds 20 patents. His research interests include robot control, design of actuators, design and control of high-speed machines, microelectromechanical systems (MEMS) 3-D assembly, micromanipulation robot, and MEMS robotic task execution. He received two National Science and Technology Awards Grade II and three Provincial Science and Technology Prizes Grade I.

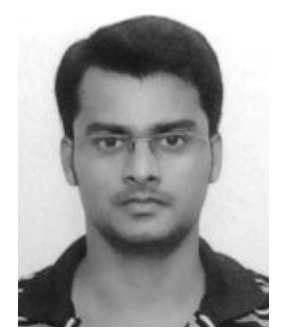

Suchith C. Menon received the B.Tech. degree in electronics engineering from the S. V. National Institute of Technology, Surat, India, in 2009, and the M.Sc. degree in electrical and computer engineering from the National University of Singapore, Singapore, in 2014. He is currently an Electronics Engineer in Singapore, and is keenly interested in analog, digital, and mixed-signal circuit design.

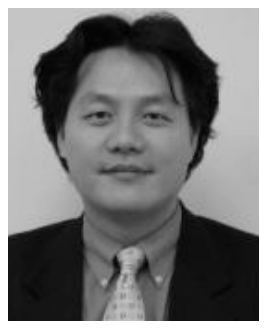

Chengkuo Lee (S'93-M'96) received the M.S. degree in materials science and engineering from National Tsing Hua University, Hsinchu, Taiwan, in 1991, the M.S. degree in industrial and system engineering from Rutgers University, New Brunswick, NJ, USA, in 1993, and the $\mathrm{Ph} . \mathrm{D}$. degree in precision engineering from the University of Tokyo, Tokyo, Japan, in 1996. He was a Foreign Researcher with the Nanometerscale Manufacturing Science Laboratory, Research Center for Advanced Science and Technology, University of Tokyo, from 1993 to 1996. He was with the Technical Engineering Laboratory, Advanced Industrial Science and Technology, Ministry of International Trade and Industry of Japan, as a Japan Science and Technology Research Fellow, in 1996. Thereafter, he was a Senior Research Staff Member with the Microsystems Laboratory, Industrial Technology Research Institute, Hsinchu. In 1997, he joined the Metrodyne Microsystem Corporation, Hsinchu, and established the Microelectromechanical Systems (MEMS) Device Division and the first Micromachining Laboratory for commercial purposes in Taiwan. He was the Manager of the MEMS Device Division from 1997 to 2000. He was an Adjunct Assistant Professor with the Department of Electrophysics, National Chiao Tung University, Hsinchu, in 1998, and an Adjunct Assistant Professor with the Institute of Precision Engineering, National Chung Hsing University, from 2001 to 2005. He co-founded Asia Pacific Microsystems, Inc. (APM), Hsinchu, in 2001, where he became the Vice President of Research and Development. In 2005, he became the Vice President of the Optical Communication Business Unit and the Special Assistant to the Chief Executive Officer in charge of international business and technical marketing for MEMS foundry service with APM, one of the top 30 MEMS manufacturers in the world. From 2006 to 2009, he was a Senior Member of the Technical Staff with the Institute of Microelectronics, Agency for Science, Technology and Research, Singapore. $\mathrm{He}$ is currently an Associate Professor with the Department of Electrical and Computer Engineering, National University of Singapore, Singapore. He has co-authored a book entitled Advanced MEMS Packaging (McGraw-Hill, 2010). He has contributed more than 230 international conference papers and extended abstracts, and 170 peer-reviewed international journal articles, and holds nine U.S. patents in MEMS, nanoelectromechanical systems, metamaterials, nanophotonics, and nanotechnology. 